\title{
Insights into the distinct roles of MMP-11 in tumor biology and future therapeutics (Review)
}

\author{
XU ZHANG ${ }^{*}$, SHUAI HUANG ${ }^{*}$, JUNCHAO GUO, LI ZHOU, LEI YOU, TAIPING ZHANG and YUPEI ZHAO \\ Department of General Surgery, Peking Union Medical College Hospital, Chinese Academy \\ of Medical Sciences/Peking Union Medical College, Beijing 100730, P.R. China
}

Received December 19, 2015; Accepted January 21, 2016

DOI: $10.3892 /$ ijo.2016.3400

\begin{abstract}
The biological processes of cancer cells such as tumorigenesis, proliferation, angiogenesis, apoptosis and invasion are greatly influenced by the surrounding microenvironment. The ability of solid malignant tumors to alter the microenvironment represents an important characteristic through which tumor cells are able to acquire specific functions necessary for their malignant biological behaviors. Matrix metalloproteinases (MMPs) are a family of zincdependent endopeptidases with the capacity of remodeling extracellular matrix (ECM) by degrading almost all ECM proteins, which plays essential roles during the invasion and metastasis process of solid malignant tumors, including allowing tumor cells to modify the ECM components and release cytokines, ultimately facilitating protease-dependent tumor progression. MMP-11, also named stromelysin-3, is a member of the stromelysin subgroup belonging to MMPs superfamily, which has been detected in cancer cells, stromal cells and adjacent microenvironment. Differently, MMP-11 exerts a dual effect on tumors. On the one hand MMP-11 promotes cancer development by inhibiting apoptosis as well as enhancing migration and invasion of cancer cells, on the other hand MMP-11 plays a negative role against cancer development via suppressing metastasis in animal models. Overexpression of MMP-11 was discovered in sera of cancer patients compared with normal control group as well as in multiple tumor tissue specimens, such as gastric cancer, breast cancer, and pancreatic cancer. At present, some
\end{abstract}

Correspondence to: Professor Junchao Guo or Professor YupeiZhao, Department of General Surgery, Peking Union Medical College Hospital, Chinese Academy of Medical Sciences/Peking Union Medical College, No. 1 Shuaifuyuan Wangfujing, Dongcheng, Beijing 100730, P.R. China

E-mail: gjcpumch@163.com

E-mail: zhao8028@263.net

*Contributed equally

Key words: Matrix metalloproteinase-11, cancer, tumorigenesis, metastasis, diagnosis, future therapeutics evidence supports that MMP-11 may work as a significant tumor biomarker for early detection of cancer, tumor staging, prognostic analysis, monitoring recurrence during follow-up and also a potential target for immunotherapy against cancer. In view of the importance of MMP-11 in modifying tumor microenvironment and potent antitumoral effects on solid tumors, there is an urgent need for a deeper understanding of how MMP-11 modulates tumor progression, and exploring its potential clinical application.

\section{Contents}

1. Introduction

2. MMP-11 and malignant solid tumors

3. Dual functions in tumorigenesis and cancer progression

4. Potential roles in cancer diagnosis, staging and treatment

5. Conclusions and perspectives

\section{Introduction}

According to recent cancer statistics data, cancer incidence has been increasing steadily in the past 10 years due to a growing and aging population, while the mortality obviously decreased as a result of earlier detection and advances in treatment (1). Therefore, understanding the mechanisms of tumorigenesis and recurrence is urgent to cut the cost of 'cancer survivors' and improve their life quality. First, it is important to design treatment strategies that will minimize the chance of occurrence and relapse in these patients. As we know, almost all solid tumors are composed of tumor cells, a variety of different non-tumor cells, defined as tumor stromal cells including endothelial cells, fibroblasts, and inflammatory cells, various cytokines, and finally their skeleton ECM $(2,3)$. All of these cells, cytokines and ECM are tightly linked and interact with each other dynamically, constituting the tumor microenvironment. It has been discovered that the tumor microenvironment has a great effect on tumorigenesis by transforming epithelial cells and changing their aptitude to give rise to malignant tumors $(2,3)$. Moreover, as for tumor progression, the complex microenvironment can affect tumor cell invasion and metastasis ability through promoting angiogenesis, recruitment of reactive stromal fibroblasts, 
immunocyte infiltration, extra production of proteolytic enzymes, and modifying the structure of ECM $(2,3)$.

Normally, ECM works as the obstacle for cell movement except during certain processes such as tissue healing and remodeling, inflammation, and neoplasia. Tumor growth and metastasis depend on the cell-cell and cell-matrix interactions and also modification of the ECM (4). In order to invade the adjacent normal parenchyma and metastasize to distant organs, surrounding ECM need to be degraded (5). Numerous evidence supports that the capacity of remolding tumor stroma is rendered by a group of molecules, including cysteine proteases, serine proteases, and matrix metalloproteinases. The interactions between tumor cells and their microenvironment reveal the key role of matrix metalloproteinases (MMPs) during the process of tumorigenesis.

Matrix metalloproteinases are zinc-dependent endopeptidases with a tremendous capacity of degrading ECM proteins, including 25 members in human. According to structural domains or corresponding specific substrates, MMPs are classified into 4 subgroups: collagenases, gelatinases, stromelysins, and membrane type MMPs (6). Functions of MMPs are fundamentally associated with matrix remodeling, such as breakdown of ECM proteins and cleavage of cell surface receptors, especially associated with cancer cell proliferation, invasion, angiogenesis and metastasis $(7,8)$. MMPs play significant roles in cancer invasion, metastasis, and angiogenesis also through their direct impact on cell behavior such as promoting growth of metastasized tumor cells, increasing motility of epithelial cells and inhibiting apoptosis.

Associations among MMP expression, advanced stages and poor clinical outcome have been observed in various types of cancer. On the other hand, MMPs may act in an indirect way. During invasion and metastasis process, degradation of ECM and basement membrane may further promote the release and activation of ECM-bound cytokines. Furthermore, ECM fragment, the products of degradation, also facilitated cell growth, motility and angiogenesis process (9). The expression and activity of these extracellular enzymes are precisely regulated at different levels. Most MMPs are produced initially as inactive proenzymes, and then processed to active forms via proteolytic cleavage. Their biological activity is also controlled by a family of natural tissue inhibitor proteins specific for MMPs (TIMPs). The imbalance between MMPs and TIMPs is implicated in various pathological tissue remodeling processes, especially during cancer progression and metastasis $(10,11)$.

The invasive nature of malignant tumors largely contributes to high mortality and poor prognosis of malignant solid tumors, which is closely associated with MMPs. Targeting MMPs could work as an optional therapeutic approach for cancer therapy. This review discusses the functions of a special matrix metalloproteinase, MMP-11, in solid tumors and its potential as a biomarker in the detection and a therapeutic target in the treatment of cancer.

\section{MMP-11 and malignant solid tumors}

Human MMP-11 (stromelysin-3) is a member of the stromelysin subgroup (6), which was first identified in the stromal cells of breast carcinoma (12). Different from other members of MMPs family, it has some unique characteristics and functions. First, it is intracytoplasmically processed and secreted as an active enzyme, whereas most MMPs are secreted as inactive zymogens (13). Second, MMP-11 does not appear to hydrolyze classical MMP-affected substrates such as laminin, fibronectin, and elastin. Instead, MMP-11 has been found to have a strong activity accelerating the degradation of serine protease inhibitor $\alpha 1$-antitrypsin, insulin-like growth factor binding protein-1 (IGFBP-1) (14), and process the capability of inducing the dedifferentiation of adipocytes and desmoplastic reaction surrounding the cancer stroma through cleavage of collagen VI $(15,16)$. These special properties suggest MMP-11, compared with other MMPs members, have a distinct role in tumor development and progression.

Similar to some members of MMPs, MMP-11 expression is found upregulated in sera of cancer patients as well as in the specimens of solid tumor tissues by immunohistochemistry, but almost absent in normal tissues. Overexpression of MMP-11 has been identified in various types of human cancers, such as oral cancer $(17,18)$, desmoid tumors (19), nonsmall cell lung cancer (20), esophageal adenocarcinoma (21), pancreatic adenocarcinoma (22), aggressive meningioma (23), colon cancer and ovarian carcinoma $(24,25)$. Apart from in invasive primary carcinomas, MMP-11 was found expressed and activated in lymph nodes and distant metastatic lesion as well, but rarely in sarcomas and other non-epithelial malignancies $(26,27)$.

MMP-11 was initially considered expressed in tumor stroma by fibroblasts surrounding tumor cells instead of by tumor cells themselves (28). However, other studies present evidence that both tumor cells and stromal cells surrounding them express MMP-11 (22,29) (Fig. 1). Cell-to-cell interactions between tumor cells and their tumor-associated fibroblasts were found to facilitate expression and activation of MMPs in tumor cells, promoting invasion and angiogenesis (30). Some prognostic factors in breast cancer including p53 expression, ER and HER2 expression, central tumor fibrosis are found to be correlated with MMP-11 expression in both tumors and stromal fibroblasts (31). These findings imply that MMP-11 is probably involved in certain signaling transduction pathways or unknown biological tumor behavior, indicating a more complex role in cancer development than its proteolysis activity.

In the following, we comprehensively summarize existing available findings on the MMP-11 expression pattern in various human cancers, as well as corresponding functions on the malignant biological properties of tumor cells.

Breast cancer. One of the main characteristics of breast cancer is its significantly high capacity of invasion and metastasis (32). Current evidence reveals that this property largely derives from interactions between cancer cells and ECM involved with MMPs and heparanase. MMP-11 was originally discovered due to its high expression in a c-DNA library established from a human breast cancer biopsy (12), in which expression of MMP-11 was found significantly upregulated by 12.45 - to 50.45 -fold in human breast cancer tissue samples compared with normal controls using Realtime RT-qPCR (33). Additionally, circulating free MMP-11 and corresponding spontaneous antibodies were also detected in the sera of breast cancer patients (34). 


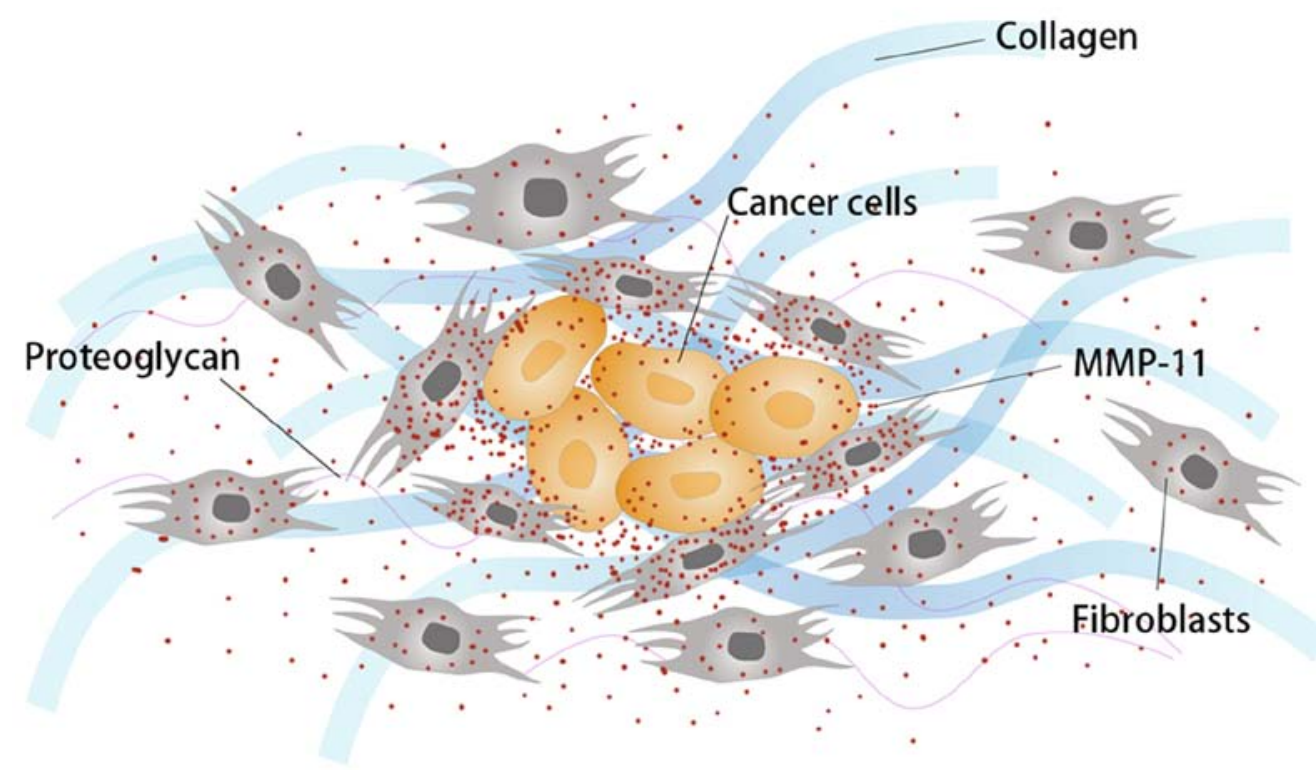

Figure 1. The expression pattern of MMP-11 in cancer microenvironment. MMP-11 is expressed by both fibroblasts in cancer stroma and cancer cells themselves. In addition, as an important component of cancer microenvironment, MMP-11 is secreted into microenvironment affecting the structure of ECM and promote the tumoregenesis and progress of cancer.

Gastric cancer. Approximately $70 \%$ of patients presenting with gastric cancer have a locally advanced and metastatic disease at the time of diagnosis followed by relatively poor prognosis, missing the possibility of radical surgical resection (35). Endogenous expression of MMP-11 in gastric cancer cells has been confirmed by western blotting and IHC staining (14). Compared with nonmalignant tissues, MMP-11 expression was significantly higher in gastric cancer specimens at both transcriptional and protein levels (36). Consistent with the expression pattern in tissues, the serum levels of MMP-11 protein were also higher in patients with advanced gastric adenocarcinoma than in healthy controls (37).

Colorectal cancer. Colorectal cancer is one of the most common human malignant neoplasms. Complications from distant metastases rather than the primary tumor itself often contribute to the death of patients in most cases. Barrasa et al (29) demonstrated that MMP-11 can be detected not only in stromal cells, but also in three different colon adenocarcinoma cell lines from epithelial origin. Of note, the expression of MMP-11 in one identical individual was finally confirmed with different patterns among normal colorectal mucosa tissues, primary colorectal cancerous tissues, and metastatic sites. The mRNA expression levels of MMP-11 in liver metastatic lesions were significantly lower compared with matched primary colon cancer tissues (38). Nevertheless, a higher expression of MMP-11 was seen in lymph node metastatic sites by immunohistochemistry (39). We can speculate that MMP-11 may play different roles in multiple subgroups of metastatic cancer cells which are genetically different from primary cells.

Pancreatic cancer. The difficulties in early detection, extremely high mortality and poor prognosis characterize pancreatic cancer. Surgery, probably the only potential option to cure, is confined when significant invasion of large peritumoral vessels and distant metastases exist, which is common for most pancre- atic cancer patients (40). More than $80 \%$ of pancreatic cancer specimens reveal strong signals for MMP-11 located in the epithelial cancer cells as well as in stromal cells surrounding them, yet MMP-11 has not been detected in mesenchymal or epithelial cells of normal pancreatic tissues (22).

Cervical cancer. Similarly, MMP-11 expression was observed in the tissues of cervical carcinoma, which has also been shown to be correlated with the development of malignancy. No MMP-11 expression was detected in normal cervical mucosa tissues, while it can be detected in approximately $50 \%$ of dysplasia, $70 \%$ of carcinoma in situ, and $100 \%$ of invasive carcinomas (41). This finding reflects that MMP-11 participates in a cancer-specific development, which needs more in-depth exploration.

\section{Dual functions in tumorigenesis and cancer progression}

While the catalytic activity of MMPs is responsible for the degrading of ECM components, which is naturally essential for promoting tumor invasion and metastasis, multiple studies have demonstrated other roles played by MMPs that are independent of proteolysis. These findings indicate more complex and even paradoxical functions of MMPs in tumorigenesis and cancer progression, possibly through interacting with different signaling pathways. The roles of MMP-11 in promoting cancer cell tumorigenesis, proliferation, and invasion have been demonstrated in many studies, which are shared by other members of MMPs family. However, it is notable that a study using MMP11-deficient transgenic mice showed unexpected dual functions of MMP-11. Overexpression of MMP-11 was found to increase the capacity of tumorigenesis in early stage, while on the other hand the metastatic ability of tumor cells are inhibited in advanced stage (42). The discrepancy whether MMP-11 during tumor development is a tumor enhancer or repressor needs clarification (Table I). 


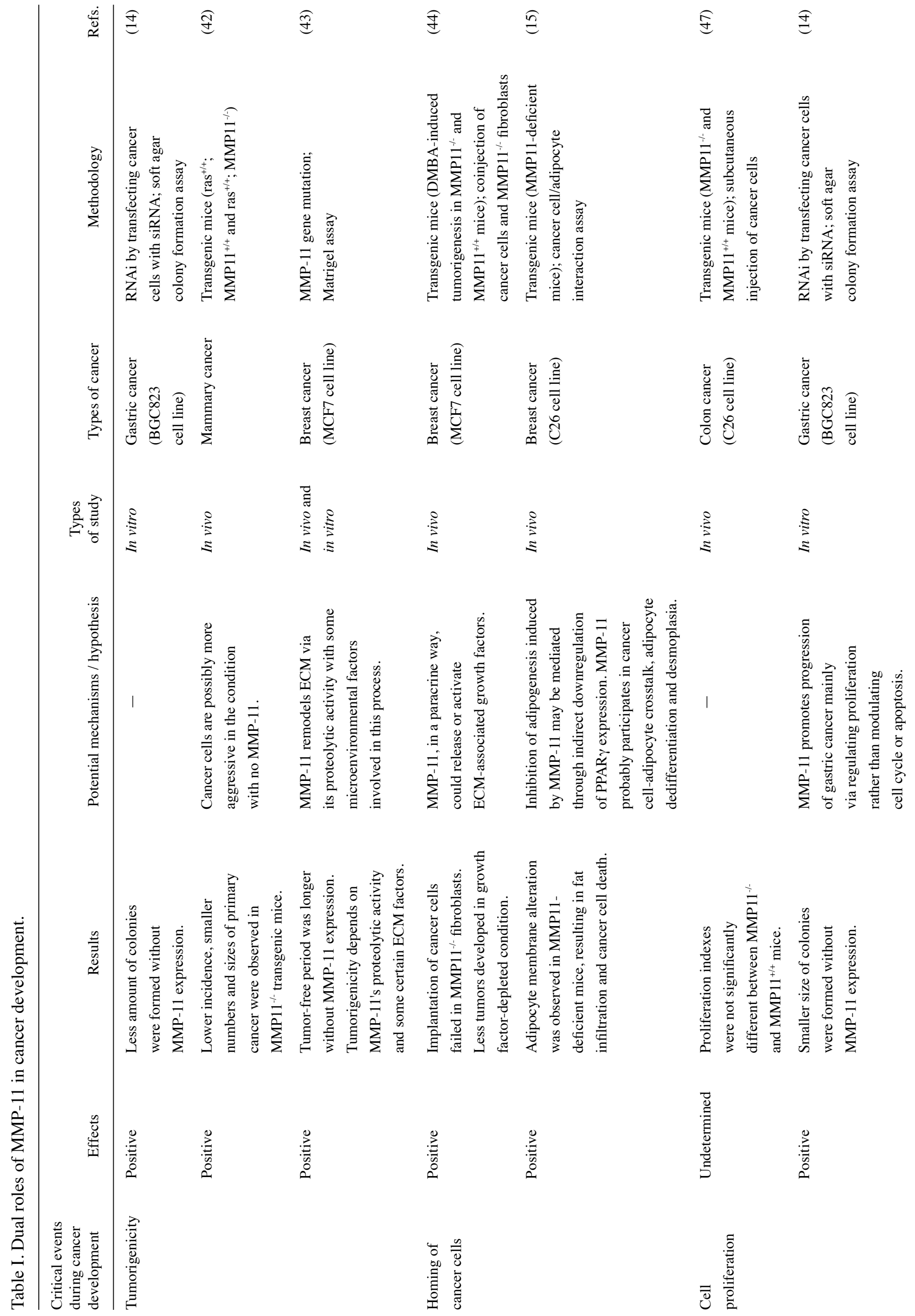




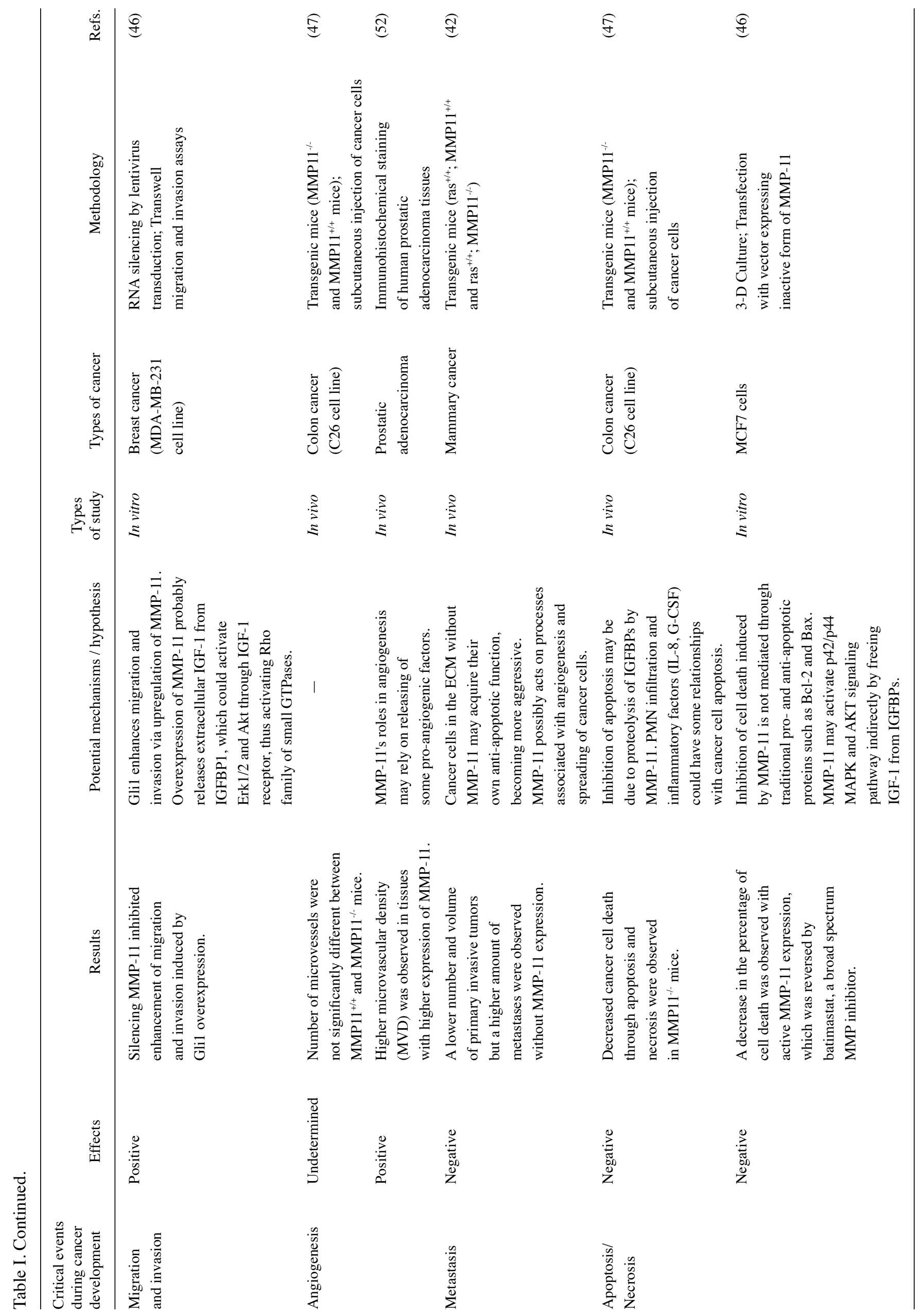


As a tumor enhancer. The tumor enhancer role of MMP-11 was considered to be natural due to its exclusive expression in most tumor tissues. Associations between MMP-11 overexpression and advanced clinicopathological staging as well as poor prognostic outcomes were also revealed.

Current evidence supports the positive effect of MMP-11 on tumorigenesis at a relatively early stage of cancer development. Downregulation of MMP-11 expression via RNA interference elevated tumorigenicity of cancer cells, leading to inhibition of tumor growth and colony formation in gastric cancer cell lines (14). In vivo, tumor-free period appeared to be longer in nude mice injected with mixed MMP11-knockdown cancer cells and complete matrigel simulating microenvironment when compared with the control group (43). However, MMP-11 seemed to lose its ability to promote tumorigenicity when MMP11-expressing cancer cells were injected with matrigel devoid of some necessary low-molecular-weight proteins instead of complete matrigel, indicating MMP-11 may affect tumorigenesis by remodeling ECM directly and freeing some necessary extracellular growth factors existing in microenvironment indirectly (43). Results from transgenic mouse models were consistent with in vitro trials. Compared with wild-type $\left(\mathrm{MMP}_{11^{+/+}}\right)$mice, MMP-11-deficient $\left(\mathrm{MMP} 11^{-/}\right)$transgenic mice presented with lower tumor incidence rate, decreased numbers, and smaller sizes of primary cancer. Furthermore, longer tumor-free survival and longer delay between the first hit of oncogene ras activation and the primary tumor appearance were observed in MMP-11-deficient mice (42).

In early stages of tumorigenesis, cancer cells are supposed to be compatible with other components in the tissue and sustained by the microenvironment. Tumor-associated fibroblasts that express MMP-11 were shown to promote homing of malignant epithelial cells. MMP11-deficient fibroblasts lost the ability to promote implantation of cancer cells in mice (44). Furthermore, MMP-11 was also found as a potent negative regulator of adipogenesis, suppressing adipocyte infiltration and cancer cell death by interfering with cancer cell-adipocyte crosstalk during tumor invasion (15).

The roles of MMP-11 in migration and invasion of cancer cells was first explained by Kwon et al (45), who found that Gli1, an established oncogene, enhanced migration and invasion via upregulation of MMP-11. Anti-apoptosis is also a basic function of MMP-11 as a tumor enhancer. MMP-11 was observed to increase survival of cancer cells in three-dimensional matrigel culture, which was reversed by batimastat, a broad spectrum MMP inhibitor. This MMP-11-mediated cell survival was found accompanied by activation of $\mathrm{p} 42 /$ p44 MAPK and AKT after analyzing apoptosis-associated proteins (46). In MMP11 ${ }^{-/-}$mice, decreased cancer cell death contributes to increased tumorigenesis rate, probably with the assistance of polymorphonuclear (PMN) infiltration and inflammatory factors (47).

The role of MMP-11 in cell proliferation remains undetermined. The MMP-11 knockdown cells by using RNAi technology exhibited significantly decreased growth ability with no remarkable change in the distribution of different phases in cell cycle (14). However, proliferation indexes were not significantly different between $\mathrm{MMP}_{1} 1^{-/}$and $\mathrm{MMP} 11^{+/+}$ mice (47). More in vitro and in vivo studies are needed to validate the effect of MMP-11 on cell proliferation.
As a tumor repressor. MMPs may also be involved in tumor repression under certain conditions. In vivo mouse experiments have shown that MMP inhibitors might promote metastasis (48). Results of the first clinical trials with broad-spectrum MMPs inhibitors in cancer therapy were disappointing; no significant therapeutic benefit (49), but rather poorer patient survival was established $(50,51)$. We consider that possibly the MMP members actually function using an as yet unknown mechanism.

Unlike several MMPs that have been shown to favor angiogenesis, MMP-11 in angiogenesis remains undetermined. Though MMP-11 is found unable to enhance angiogenesis in an animal-based tumorigenesis model (47), immunochemical staining of human prostatic adenocarcinoma tissues revealed that microvascular density (MVD) was significantly associated with the MMP-11 expression levels (52). Although longer tumor-free survival, fewer and smaller primary tumors were observed in MMP11-deficient mice (42), a systemic search for hidden lung metastases revealed a significant higher number of metastases in the absence of MMP-11, indicating an increased possibility for hematogenous dissemination (53). Similar to previous reports, the number of lung metastases developed in MMP-11-deficient mice was 2-3-fold higher than in wild-type mice using micro-CT and histological analysis, while primary mammary tumors were comparatively fewer and smaller (54).

Crosstalk with other tumor-related molecules. As mentioned above, there still remains controversy regarding the relationships between MMP-11 expression and malignant biological properties in various malignant tumors. How MMP-11 acts in the process of cancer development still remain unclear. It is assumed that MMP-11 plays an extremely complex role and are involved in various signal pathways, such as MAP kinase, Wnt, and PI3-kinase, and/or MMPs inducers, for example CD147 $(31,55)$, displaying variant biological effects. On the contrary, MMP expression was found to be modulated at different levels by a series of growth factors, hormones and cytokines $(56,57)$. Selvey et al (58) observed that MMP-11 expression was markedly amplified after treatments with IL-1 $\beta$, IL-2, TGF- $\beta 1$, fibronectin and collagen V. This overexpression of MMP-11 was inhibited by progesterone, a potent inhibitor of TGF $\beta 1$ (59). Retinoids were found acting predominantly at a post-transcriptional level to inhibit MMP-11 expression in human pancreatic carcinoma cell lines (22). Interactions among various MMPs also constitute a complex network. Membranous MMP-14 could hydrolyze and inactivate MMP-11, thus restricting its functions spatially (60). It is also found in renal cell carcinoma that microRNA-145 could suppress cell proliferation, migration and invasion by directly downregulating the expression of MMP-11 (61). In conclusion, the regulatory pathways of MMP-11 are complicated and need to be further explored.

Insulin-like growth factor-1 (IGF-1) is an important modulator of cancer metastasis. Upregulation of IGF-1 was reported in invasive and metastatic cancer, playing a key role during tumor proliferation and progression (62). In addition, IGF-1 receptor blockade could promote radiation- and chemotherapyinduced apoptosis in tumor-bearing mice (63). Some studies showed that interactions between MMP-11 and IGF-1 are closely related (64). Zhao et al (36) further demonstrated that 


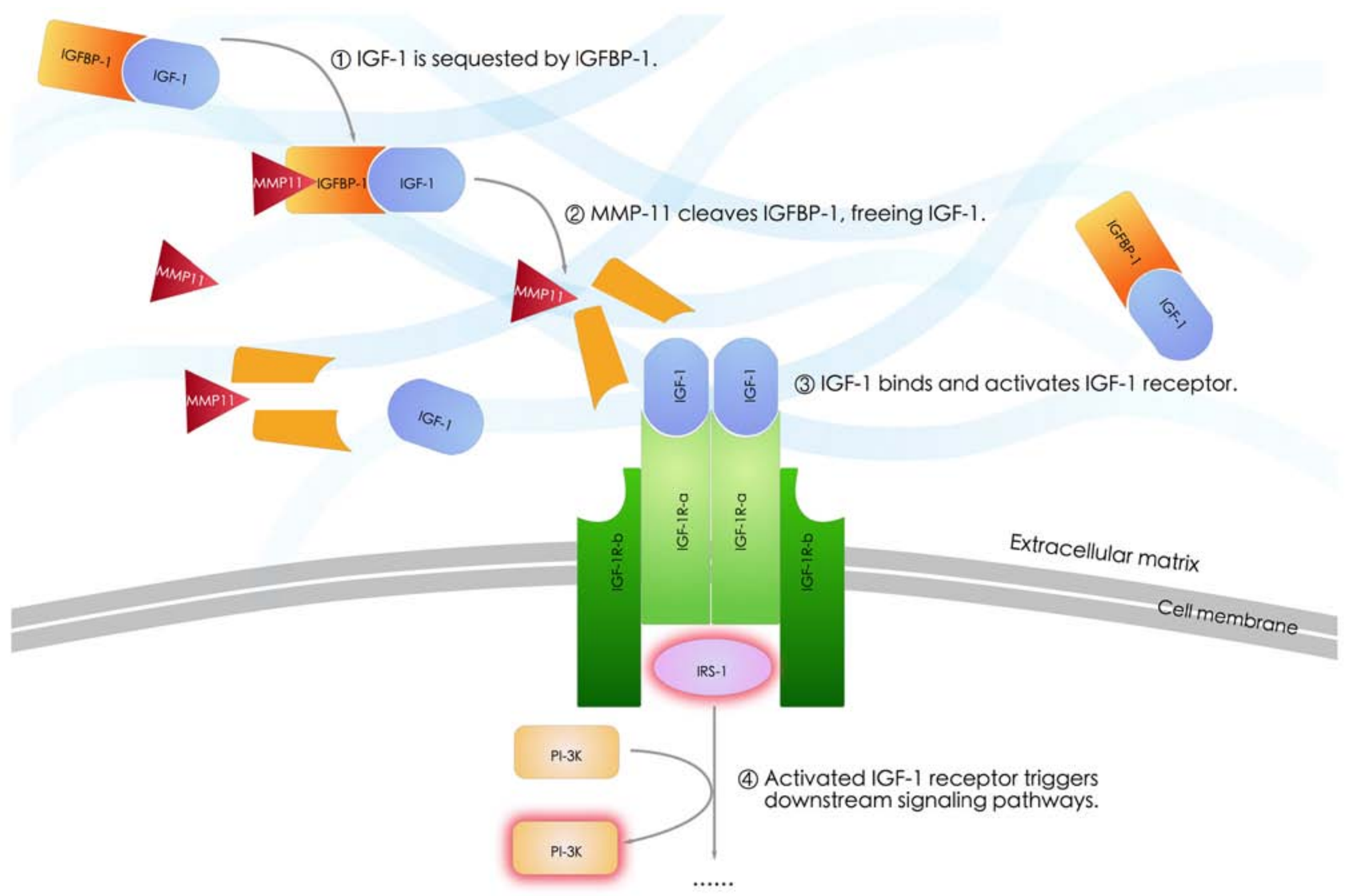

Figure 2. The Role of MMP-11 in insulin-like growth factor-1 (IGF-1) signaling pathway. IGF-1, located in cancer cell microenvironment, is an important effector for facilitating cell growth and motility. While, the IGF-1's biological activity was neutralized by its specific binding protein IGFBP-1, which could be cleaved by MMP-11 as a substrate. Extra free IGF-1 in microenvironment significantly bind to its receptors (IGF-1 R) on the cell membrane, activating the IGF-1-mediated signaling pathway. Among the downstream cascade of reactions, the most relevant is the insulin receptor substrate-1 (IRS-1)-PI-3K signal pathway.

MMP-11 expression was positively correlated with increased expression of IGF-1. It is implied that MMP-11 protein is capable of cleaving insulin-like growth factor binding protein 1 (IGFBP-1), thereby freeing IGF-1 and activating IGF-1 signaling cascade, triggering down-stage cascade reactions such as activation of PI-3K (55,65); (Fig. 2).

CD147 is a plasma membrane glycoprotein, which is highly located on the surface of many malignant tumor cells and facilitates tumor cells or stromal fibroblasts to generate MMPs (12). Associations between CD147 expression with unfavorable prognosis as well as its pro-angiogenesis effect have been shown in breast cancer and glioblastoma $(66,67)$. A recent study also demonstrated that both CD147 and MMP-11 were involved in the progression of colorectal cancer, which were proven to be independent prognostic factors (68). As for potential mechanisms, increased CD147 expression in hepatocarcinoma cells was observed to significantly promote downregulation of MMP-11 and VEGF-A at both mRNA and protein levels (69). Additionally, glycosylation status of CD147 is associated with the expression level of MMP-11, which affects the adhesive and invasive ability of tumor cells in vitro (70).

Altogether, the exact roles of MMP-11 in tumorigenesis and cancer progression seem to be complex and controversial, depending on the location and status of solid tumors. Current evidence demonstrates that it could facilitate tumorigenesis, migration and invasion, homing of malignant epithelial cells, and anti-apoptosis effect as a tumor enhancer, while inhibiting metastatic development as a possible tumor repressor.

\section{Potential roles in cancer diagnosis, staging and treatment}

It has been shown that MMPs take part in nearly every critical event during tumor development. Also, ample evidence demonstrates that the members of MMPs could work as a possible indicator for screening cancer at an early stage, monitoring tumor progression and predicting outcomes. For example, MMP-2, MMP-7, and MMP-9 have been reported to be related with the progression and prognosis of endometrial carcinoma (71), colorectal cancer $(72)$, gastric cancer $(73,74)$, and breast cancer (75). Herein, we present some recent studies supporting the clinical value of MMP-11 as a promising biomarker, prognosis predictor or a potential target for immunotherapy against cancer.

Early diagnosis. Currently, invasion and metastasis status at the early stage are major obstacles for cancer management such as pancreatic cancer and gastric cancer. Thus, finding a potent biomarker for clinical diagnosis is the premise for effective treatment to malignant solid tumors. We have made great efforts in exploring valid markers with high sensitivity and 
specificity. In order to assist in early diagnosis and making the most optimal treatment strategies, the potential marker must be easily acquired, for example through serum or specimens.

MMP-11, which is a secreted protein, not only takes effect in the cytoplasm, but is secreted to the extracellular space as an important component in the microenvironment, affecting both the tumors and the ECM. MMP-11 is highly expressed in cancer tissues compared with adjacent normal tissues. Some evidence indicates that MMP-11 plays significant roles in early tumorigenesis, suggesting its potential to be a novel biomarker for early detection of cancer. Serum MMP-11 efficacy of diagnosis has been proved in several studies. It has been indicated that serum levels of MMP-11 were significantly elevated in gastric cancer patients compared with normal controls. Additionally, MMP-11 was also observed elevated in the sera of intestinal metaplasia and dysplasia patients (76). Sensitivity for diagnosis of gastric cancer using MMP-11 was higher when compared with other traditional tumor markers, such as CA199, CEA, CA242 and MMP-9 (76). In another study, a certain cut-off value of MMP-11 protein could reach the optimal sensitivity and specificity according to ROC analysis for diagnosis of gastric adenocarcinoma, 94\% and $93.7 \%$, respectively (37).

Staging and prediction of prognosis. Identification of prognostic indicators has always been one of the research hotspots in cancer research due to the aim of making appropriate treatment strategies. The expression levels of MMP-11 could be used to identify patients at greater risk for cancer recurrence in breast carcinoma (77), pancreatic tumors (78), and colon cancer (79). Higher levels of MMP-11 expression were observed in poorly differentiated high-grade thyroid carcinoma (80) and in breast carcinoma (81), correlating with less survival time among patients with breast, non-small cell lung cancer and colon cancer $(77,82)$.

Overexpression of MMP-11 was reported to be associated with several clinicopathological characteristics, such as poor differentiation, lymph node metastasis and lack of progesterone receptor, in oral squamous cell carcinoma (83) as well as in breast carcinoma (34). Temporally increased MMP-11 expression can be considered as an early event, prior to lymph node metastasis during breast cancer progression (34). In gastric cancer patients, some evidence shows MMP-11 is not only correlated with advanced-stage and high-grade tumors (36), but significantly associated with metastasis (76), especially for lymph node metastasis (84) rather than peritoneal seeding and distant organ metastasis (37). Pedersen et al (85) found higher expression of MMP-11 in colon carcinomas was associated with invasion depth, presence of metastasis, and poor differentiation. In addition, expression of MMP-11 and VEGF-C in colorectal adenocarcinoma is believed to be an important index for predicting distant metastases and clinical stages (86).

The prognostic significance of MMP-11 expression is also reported in gastric carcinoma, indicating its role in predicting outcomes and monitoring recurrence during follow-up. The 5-year survival rates of patients with high expression of both MMP-11 and IGF-1 was significantly lower compared to the patients with low expression levels of both MMP-11 and IGF-1 (36), which proved MMP-11 and IGF-1 to be independent prognostic factors in patients with gastric carcinoma. A study concerning gastric adenocarcinoma revealed that patients with low expression levels of MMP-11 had longer median survival time and one-year survival rate than those with high levels, while the median TTP time was not significantly different from those with high levels of MMP-11 (37). Similarly in prostate cancer patients, high MMP-11 expression was significantly correlated with poor differentiation degree in Gleason grading, late tumor stage, and positive bone metastasis. Overall survival time of patients with higher levels of MMP-11 was significantly shorter than those with low levels (34). Furthermore, a recent study by Eiró et al (87) found that MMP-11 expression by mononuclear inflammatory cells (MICs) was a potent prognostic factor for predicting outcomes of patients with primary ductal invasive breast tumors. This finding implies multiple functions of MMP-11 in various types of cells in the tumor microenvironment, which may exert its influence upon cancer development on different levels.

A promising therapeutic target. Stromal cells in tumor tissues are genetically more stable compared with cancer cells, but differ from their counterparts in normal tissues for types and levels of certain proteins $(10,88)$. This feature indicates that stromal antigens may also work as antitumoral targets. Immunotherapy regimens targeting stromal antigens may be shared by several tumor types as stromal antigens are often expressed by a broad spectrum of solid tumors (89). Several preclinical and clinical studies have shown the possibility that targeting tumor stroma is a promising therapeutic target. At present, targets of immune interventions include cancer-associated fibroblasts, infiltrating macrophages/histiocytes, and tumor endothelial cells. Special antigens such as carbonic anhydrase IX or fibroblast activation protein (FAP) $\alpha$ suggest that vaccination against stromal antigens is a feasible therapeutic approach (90). Immunologic targeting of MMPs has been demonstrated by several studies. A vaccine against MMP-2 was reported with an effective antitumoral function, prolonging the survival time of cancerbearing mice (91). MMP-7 was also identified as a broadly expressed tumor-associated antigen target, which could be a candidate for antitumoral vaccine (92). Moreover, a longer progression-free survival (PFS) was observed in patients with recurrent and progressive glioblastoma after a phase II clinical trial, who were administered marimastat (a broad spectrum MMPs inhibitor) in conjunction with an additional cytotoxic agent following standard radiotherapy (93). These results support that MMPs are promising targets for antigen-specific immunotherapy.

Considering MMP-11 is expressed exclusively in most primary solid cancer tissues and metastatic lesions, it is an ideal antigen target for immunotherapy. The therapeutic potency of MMP-11 was also demonstrated in some studies. Peruzzi et al (89) discovered that a genetic vaccine against MMP-11 based on DNA electro-gene-transfer technology was able to break immune tolerance and exert anti-tumor effects in a colon-adenocarcinoma mouse model. This vaccine significantly reduced tumor formation at all precancerous stages, and inhibited tumor progression by reducing the number of lesions at later periods. Therefore, targeting MMP-11 therapy may be potentially efficient in controlling disease progression. 


\section{Conclusions and perspectives}

A number of researches have shown that MMPs not only participate in tumor invasion and metastasis or the late stages of the carcinogenesis but are also implicated in early stages of tumorigenesis in both favorable and unfavorable manners. MMPs are a diverse group of enzymes with high heterogeneity, exerting various functions based on different types and stages of solid tumors. Thus the clinical use of different MMP subgroups and their inhibitors must take into consideration of the type and stage of tumor. According to current theory, MMPs take effects mainly through reconstructing the tumor microenvironment, so MMP inhibitors would be more effective in certain type of solid tumors where stroma plays an important role, such as pancreatic cancer.

MMP-11, a member of MMP family, comes under the spotlight due to its distinct characteristics. Overexpression of MMP-11 can be detected among various malignant cancers including breast cancer, colorectal cancer, gastric cancer, and pancreatic cancer. Originally, the role of MMP-11 in tumorigenesis and cancer progression was regarded as a tumor-facilitating factor by promoting colony formation, remodeling extracellular matrix and suppressing apoptosis, which is in accord with the proteolysis activity of MMPs. However, recent evidence demonstrated MMP-11 may work as a tumor repressor by inhibiting metastasis in certain tumors. Therefore, we can preliminarily speculate that MMP-11 may play a dual role, not only a tumor enhancer, but a repressor during cancer development. Therefore, more efforts are required to clarify the exact mechanisms. As MMP-11 expression is upregulated with the progress of cancer development and exclusively located at cancer tissues compared to normal controls, it is proved to be a potential biomarker for prognosis analysis. The expression levels of MMP-11 are also found to be significantly associated with some important clinicopathological characteristics. Additionally, considering the relatively high specificity of MMP-11 expression in cancer tissues, some initial trials evaluating the effects of anti-MMP-11 immunotherapy suggest that MMP-11 is a promising therapeutic target for cancer treatment. While, limited by the single center study and relative small number of patients, more well-designed clinical trials are needed to evaluate the treatment efficacy of MMP-11-monoclonal antibody therapy.

We have realized the importance of MMP-11 on biological behaviors of cancer development. Nevertheless, at the time of this review, no document proves an exact mechanism involved in MMP-11-participated modulation process.

Further efforts should be made to explore the comprehensive mechanisms and associated signal molecules, as well as to find a promising therapy target to reduce the pro-tumorigenesis effect of MMP-11. Moreover, the potential prognostic value of MMP-11 in the serum should be verified in large-scale multicenter clinical studies.

\section{Acknowledgements}

This work was funded by National High Technology Research and Development Program (863 Program, 2014AA020609), China.

\section{References}

1. DeSantis CE, Lin CC, Mariotto AB, Siegel RL, Stein KD, Kramer JL, Alteri R, Robbins AS and Jemal A: Cancer treatment and survivorship statistics, 2014. CA Cancer J Clin 64: 252-271, 2014.

2. Frisch SM and Francis H: Disruption of epithelial cell-matrix interactions induces apoptosis. J Cell Biol 124: 619-626, 1994.

3. Wei L and Shi YB: Matrix metalloproteinase stromelysin-3 in development and pathogenesis. Histol Histopathol 20: 177-185, 2005.

4. Wieczorek E, Jablonska E, Wasowicz W and Reszka E: Matrix metalloproteinases and genetic mouse models in cancer research: a mini-review. Tumour Biol 36: 163-175, 2015.

5. Geho DH, Bandle RW, Clair T and Liotta LA: Physiological mechanisms of tumor-cell invasion and migration. Physiology (Bethesda) 20: 194-200, 2005.

6. Motrescu ER and Rio MC: Cancer cells, adipocytes and matrix metalloproteinase 11: A vicious tumor progression cycle. Biol Chem 389: 1037-1041, 2008.

7. Vazquez-Ortiz G, Pina-Sanchez P, Vazquez K, Duenas A, Taja L, Mendoza P, Garcia JA and Salcedo M: Overexpression of cathepsin F, matrix metalloproteinases 11 and 12 in cervical cancer. BMC Cancer 5: 68, 2005.

8. Bartolomé RA, Ferreiro S, Miquilena-Colina ME, MartínezPrats L, Soto-Montenegro ML, García-Bernal D, Vaquero JJ, Agami R, Delgado R, Desco M, et al: The chemokine receptor CXCR4 and the metalloproteinase MT1-MMP are mutually required during melanoma metastasis to lungs. Am J Pathol 174: 602-612, 2009

9. Chen Y, Chen Y, Huang L and Yu J: Evaluation of heparanase and matrix metalloproteinase-9 in patients with cutaneous malignant melanoma. J Dermatol 39: 339-343, 2012.

10. Egeblad M and Werb Z: New functions for the matrix metalloproteinases in cancer progression. Nat Rev Cancer 2: 161-174, 2002.

11. Folgueras AR, Pendás AM, Sánchez LM and López-Otín C: Matrix metalloproteinases in cancer: From new functions to improved inhibition strategies. Int J Dev Biol 48: 411-424, 2004.

12. Basset P, Bellocq JP, Wolf C, Stoll I, Hutin P, Limacher JM, Podhajcer OL, Chenard MP, Rio MC and Chambon P: A novel metalloproteinase gene specifically expressed in stromal cells of breast carcinomas. Nature 348: 699-704, 1990.

13. Pei D and Weiss SJ: Furin-dependent intracellular activation of the human stromelysin-3 zymogen. Nature 375: 244-247, 1995.

14. Deng H, Guo RF, Li WM, Zhao M and Lu YY: Matrix metalloproteinase 11 depletion inhibits cell proliferation in gastric cancer cells. Biochem Biophys Res Commun 326: 274-281, 2005.

15. Andarawewa KL, Motrescu ER, Chenard MP, Gansmuller A, Stoll I, Tomasetto C and Rio MC: Stromelysin-3 is a potent negative regulator of adipogenesis participating to cancer celladipocyte interaction/crosstalk at the tumor invasive front. Cancer Res 65: 10862-10871, 2005.

16. Motrescu ER, Blaise S, Etique N, Messaddeq N, Chenard MP, Stoll I, Tomasetto C and Rio MC: Matrix metalloproteinase-11/ stromelysin-3 exhibits collagenolytic function against collagen VI under normal and malignant conditions. Oncogene 27: 6347-6355, 2008.

17. Soni S, Mathur M, Shukla NK, Deo SV and Ralhan R: Stromelysin-3 expression is an early event in human oral tumorigenesis. Int J Cancer 107: 309-316, 2003.

18. Arora S, Kaur J, Sharma C, Mathur M, Bahadur S, Shukla NK, Deo SV and Ralhan R: Stromelysin 3, Ets-1, and vascular endothelial growth factor expression in oral precancerous and cancerous lesions: correlation with microvessel density, progression, and prognosis. Clin Cancer Res 11: 2272-2284, 2005.

19. Denys H, De Wever O, Nusgens B, Kong Y, Sciot R, Le AT, Van Dam K, Jadidizadeh A, Tejpar S, Mareel M, et al: Invasion and MMP expression profile in desmoid tumours. Br J Cancer 90: 1443-1449, 2004

20. Kettunen E, Anttila S, Seppänen JK, Karjalainen A, Edgren H, Lindström I, Salovaara R, Nissén AM, Salo J, Mattson K, et al: Differentially expressed genes in nonsmall cell lung cancer: Expression profiling of cancer-related genes in squamous cell lung cancer. Cancer Genet Cytogenet 149: 98-106, 2004.

21. Hourihan RN, O'Sullivan GC and Morgan JG: Transcriptional gene expression profiles of oesophageal adenocarcinoma and normal oesophageal tissues. Anticancer Res 23 (1A): 161-165, 2003. 
22. von Marschall Z, Riecken EO and Rosewicz S: Stromelysin 3 is overexpressed in human pancreatic carcinoma and regulated by retinoic acid in pancreatic carcinoma cell lines. Gut 43: 692-698, 1998.

23. Perret AG, Duthel R, Fotso MJ, Brunon J and Mosnier JF: Stromelysin-3 is expressed by aggressive meningiomas. Cancer 94: 765-772, 2002

24. Mueller J, Brebeck B, Schmalfeldt B, Kuhn W, Graeff H and Höfler H: Stromelysin-3 expression in invasive ovarian carcinomas and tumours of low malignant potential. Virchows Arch 437: 618-624, 2000

25. Wlodarczyk J, Stolte M and Mueller J: E-cadherin, beta-catenin and stromelysin-3 expression in de novo carcinoma of the colorectum. Pol J Pathol 52: 119-124, 2001.

26. Wolf C, Rouyer N, Lutz Y, Adida C, Loriot M, Bellocq JP, Chambon $\mathrm{P}$ and Basset $\mathrm{P}$ : Stromelysin 3 belongs to a subgroup of proteinases expressed in breast carcinoma fibroblastic cells and possibly implicated in tumor progression. Proc Natl Acad Sci USA 90: 1843-1847, 1993.

27. Basset P, Okada A, Chenard MP, Kannan R, Stoll I, Anglard P, Bellocq JP and Rio MC: Matrix metalloproteinases as stromal effectors of human carcinoma progression: therapeutic implications. Matrix Biol 15: 535-541, 1997.

28. Laurell H, Bouisson M, Berthelemy P, Rochaix P, Dejean S, Besse P, Susini C, Pradayrol L, Vaysse N and Buscail L: Identification of biomarkers of human pancreatic adenocarcinomas by expression profiling and validation with gene expression analysis in endoscopic ultrasound-guided fine needle aspiration samples. World J Gastroenterol 12: 3344-3351, 2006.

29. Barrasa JI, Olmo N, Santiago-Gómez A, Lecona E, Anglard P, Turnay $\mathrm{J}$ and Lizarbe MA: Histone deacetylase inhibitors upregulate MMP11 gene expression through Sp1/Smad complexes in human colon adenocarcinoma cells. Biochim Biophys Acta 1823: 570-581, 2012.

30. Genestie C, Zafrani B, Asselain B, Fourquet A, Rozan S, Validire P, Vincent-Salomon A and Sastre-Garau X: Comparison of the prognostic value of Scarff-Bloom-Richardson and Nottingham histological grades in a series of 825 cases of breast cancer: Major importance of the mitotic count as a component of both grading systems. Anticancer Res 18: 571-576, 1998.

31. Min KW, Kim DH, Do SI, Pyo JS, Kim K, Chae SW, Sohn JH, Oh YH, Kim HJ, Choi SH, et al: Diagnostic and prognostic relevance of MMP-11 expression in the stromal fibroblast-like cells adjacent to invasive ductal carcinoma of the breast. Ann Surg Oncol 20 (Suppl 3): S433-S442, 2013.

32. DeSantis C, Ma J, Bryan L and Jemal A: Breast cancer statistics, 2013. CA Cancer J Clin 64: 52-62, 2014.

33. Fu J, Khaybullin R, Zhang Y, Xia A and Qi X: Gene expression profiling leads to discovery of correlation of matrix metalloproteinase 11 and heparanase 2 in breast cancer progression. BMC Cancer 15: 473, 2015.

34. Roscilli G, Cappelletti M, De Vitis C, Ciliberto G, Di Napoli A, Ruco L, Mancini R and Aurisicchio L: Circulating MMP11 and specific antibody immune response in breast and prostate cancer patients. J Transl Med 12: 54, 2014.

35. Lustosa SA, Saconato H, Atallah AN, Lopes Filho Gde J and Matos D: Impact of extended lymphadenectomy on morbidity, mortality, recurrence and 5-year survival after gastrectomy for cancer. Meta-analysis of randomized clinical trials. Acta Cir Bras 23: 520-530, 2008

36. Zhao ZS, Chu YQ, Ye ZY, Wang YY and Tao HQ: Overexpression of matrix metalloproteinase 11 in human gastric carcinoma and its clinicopathologic significance. Hum Pathol 41: 686-696, 2010.

37. Yan D, Dai H and Liu JW: Serum levels of MMP-11 correlate with clinical outcome in Chinese patients with advanced gastric adenocarcinoma. BMC Cancer 11: 151, 2011.

38. Asano T, Tada M, Cheng S, Takemoto N, Kuramae T, Abe M, Takahashi O, Miyamoto M, Hamada J, Moriuchi T, et al: Prognostic values of matrix metalloproteinase family expression in human colorectal carcinoma. J Surg Res 146: 32-42, 2008

39. Skoglund J, Emterling A, Arbman G, Anglard P and Sun XF: Clinicopathological significance of stromelysin-3 expression in colorectal cancer. Oncology 67: 67-72, 2004.

40. Bakkevold KE, Arnesjø B and Kambestad B: Carcinoma of the pancreas and papilla of Vater: Presenting symptoms, signs, and diagnosis related to stage and tumour site. A prospective multicentre trial in 472 patients. Norwegian Pancreatic Cancer Trial Scand J Gastroenterol 27: 317-325, 1992.

41. Rouyer N, Wolf C, Chenard MP, Rio MC, Chambon P, Bellocq JP and Basset P: Stromelysin-3 gene expression in human cancer: An overview. Invasion Metastasis 14: 269-275, 1994-1995.
42. Andarawewa KL, Boulay A, Masson R, Mathelin C, Stoll I, Tomasetto C, Chenard MP, Gintz M, Bellocq JP and Rio MC: Dual stromelysin-3 function during natural mouse mammary tumor virus-ras tumor progression. Cancer Res 63: 5844-5849, 2003.

43. Noël A, Boulay A, Kebers F, Kannan R, Hajitou A, Calberg-Bacq CM, Basset P, Rio MC and Foidart JM: Demonstration in vivo that stromelysin-3 functions through its proteolytic activity. Oncogene 19: 1605-1612, 2000.

44. Masson R, Lefebvre O, Noël A, Fahime ME, Chenard MP, Wendling C, Kebers F, LeMeur M, Dierich A, Foidart JM, et al: In vivo evidence that the stromelysin-3 metalloproteinase contributes in a paracrine manner to epithelial cell malignancy. $\mathrm{J}$ Cell Biol 140: 1535-1541, 1998.

45. Kwon YJ, Hurst DR, Steg AD, Yuan K, Vaidya KS, Welch DR and Frost AR: Gli1 enhances migration and invasion via up-regulation of MMP-11 and promotes metastasis in ERo negative breast cancer cell lines. Clin Exp Metastasis 28: 437-449, 2011.

46. Fromigue O, Louis K, Wu E, Belhacène N, Loubat A, Shipp M, Auberger P and Mari B: Active stromelysin-3 (MMP-11) increases MCF-7 survival in three-dimensional Matrigel culture via activation of p42/p44 MAP-kinase. Int J Cancer 106: 355-363, 2003.

47. Boulay A, Masson R, Chenard MP, El Fahime M, Cassard L, Bellocq JP, Sautès-Fridman C, Basset P and Rio MC: High cancer cell death in syngeneic tumors developed in host mice deficient for the stromelysin-3 matrix metalloproteinase. Cancer Res 61: 2189-2193, 2001.

48. Krüger A, Soeltl R, Sopov I, Kopitz C, Arlt M, Magdolen V, Harbeck N, Gänsbacher B and Schmitt M: Hydroxamate-type matrix metalloproteinase inhibitor batimastat promotes liver metastasis. Cancer Res 61: 1272-1275, 2001.

49. Zucker S, Cao J and Chen WT: Critical appraisal of the use of matrix metalloproteinase inhibitors in cancer treatment. Oncogene 19: 6642-6650, 2000.

50. Coussens LM, Fingleton B and Matrisian LM: Matrix metalloproteinase inhibitors and cancer: Trials and tribulations. Science 295: 2387-2392, 2002.

51. Overall CM and López-Otín C: Strategies for MMP inhibition in cancer: Innovations for the post-trial era. Nat Rev Cancer 2: 657-672, 2002.

52. Kanharat $\mathrm{N}$ and Tuamsuk P: Correlation between microvascular density and matrix metalloproteinase 11 expression in prostate cancer tissues: A preliminary study in Thailand. Asian Pac J Cancer Prev 16: 6639-6643, 2015.

53. Rio MC: From a unique cell to metastasis is a long way to go: Clues to stromelysin-3 participation. Biochimie 87: 299-306, 2005.

54. Brasse D, Mathelin C, Leroux K, Chenard MP, Blaise S, Stoll I, Tomasetto C and Rio MC: Matrix metalloproteinase 11/ stromelysin-3 exerts both activator and repressor functions during the hematogenous metastatic process in mice. Int J Cancer 127: $1347-1355,2010$.

55. Kasper G, Reule M, Tschirschmann M, Dankert N, StoutWeider K, Lauster R, Schrock E, Mennerich D, Duda GN and Lehmann KE: Stromelysin-3 over-expression enhances tumourigenesis in MCF-7 and MDA-MB-231 breast cancer cell lines: Involvement of the IGF-1 signalling pathway. BMC Cancer 7: 12, 2007.

56. Jones L, Ghaneh P, Humphreys M and Neoptolemos JP: The matrix metalloproteinases and their inhibitors in the treatment of pancreatic cancer. Ann NY Acad Sci 880: 288-307, 1999.

57. Johansson N, Ahonen M and Kähäri VM: Matrix metalloproteinases in tumor invasion. Cell Mol Life Sci 57: 5-15, 2000.

58. Selvey S, Haupt LM, Thompson EW, Matthaei KI, Irving MG and Griffiths LR: Stimulation of MMP-11 (stromelysin-3) expression in mouse fibroblasts by cytokines, collagen and co-culture with human breast cancer cell lines. BMC Cancer 4: 40, 2004

59. Itoh H, Kishore AH, Lindqvist A, Rogers DE and Word RA: Transforming growth factor $\beta 1$ (TGF $\beta 1)$ and progesterone regulate matrix metalloproteinases (MMP) in human endometrial stromal cells. J Clin Endocrinol Metab 97: E888-E897, 2012.

60. Buache E, Thai R, Wendling C, Alpy F, Page A, Chenard MP, Dive V, Ruff M, Dejaegere A, Tomasetto C, et al: Functional relationship between matrix metalloproteinase-11 and matrix metalloproteinase-14. Cancer Med 3: 1197-1210, 2014.

61. Wu D, Li M, Wang L, Zhou Y, Zhou J, Pan H and Qu P: microRNA-145 inhibits cell proliferation, migration and invasion by targeting matrix metallopeptidase-11 in renal cell carcinoma. Mol Med Rep 10: 393-398, 2014. 
62. Jiang Y, Wang L, Gong W, Wei D, Le X, Yao J, Ajani J, Abbruzzese JL, Huang S and Xie K: A high expression level of insulin-like growth factor I receptor is associated with increased expression of transcription factor Sp1 and regional lymph node metastasis of human gastric cancer. Clin Exp Metastasis 21: 755-764, 2004

63. Min Y, Adachi Y, Yamamoto H, Imsumran A, Arimura Y, Endo T, Hinoda Y, Lee CT, Nadaf S, Carbone DP, et al: Insulin-like growth factor I receptor blockade enhances chemotherapy and radiation responses and inhibits tumour growth in human gastric cancer xenografts. Gut 54: 591-600, 2005.

64. Sharma R, Chattopadhyay TK, Mathur M and Ralhan R: Prognostic significance of stromelysin-3 and tissue inhibitor of matrix metalloproteinase-2 in esophageal cancer. Oncology 67: 300-309, 2004.

65. Mañes S, Mira E, Barbacid MM, Ciprés A, Fernández-Resa P, Buesa JM, Mérida I, Aracil M, Márquez G and Martínez-A C: Identification of insulin-like growth factor-binding protein-1 as a potential physiological substrate for human stromelysin-3. J Biol Chem 272: 25706-25712, 1997.

66. Liang Q, Xiong H, Gao G, Xiong K, Wang X, Zhao Z, Zhang H and $\mathrm{Li}$ Y: Inhibition of basigin expression in glioblastoma cell line via antisense RNA reduces tumor cell invasion and angiogenesis. Cancer Biol Ther 4: 759-762, 2005.

67. Tang Y, Nakada MT, Rafferty P, Laraio J, McCabe FL, Millar H, Cunningham M, Snyder LA, Bugelski P and Yan L: Regulation of vascular endothelial growth factor expression by EMMPRIN via the PI3K-Akt signaling pathway. Mol Cancer Res 4: 371-377, 2006

68. Tian X, Ye C, Yang Y, Guan X, Dong B, Zhao M and Hao C: Expression of CD147 and matrix metalloproteinase-11 in colorectal cancer and their relationship to clinicopathological features. J Transl Med 13: 337, 2015.

69. Jia L, Cao J, Wei W, Wang S, Zuo Y and Zhang J: CD147 depletion down-regulates matrix metalloproteinase-11, vascular endothelial growth factor-A expression and the lymphatic metastasis potential of murine hepatocarcinoma Hca-F cells. Int J Biochem Cell Biol 39: 2135-2142, 2007.

70. Jia L, Zhou H, Wang S, Cao J, Wei W and Zhang J: Deglycosylation of CD147 down-regulates matrix metalloproteinase-11 expression and the adhesive capability of murine hepatocarcinoma cell HcaF in vitro. IUBMB Life 58: 209-216, 2006.

71. Honkavuori M, Talvensaari-Mattila A, Soini Y, TurpeenniemiHujanen T and Santala M: MMP-2 expression associates with CA 125 and clinical course in endometrial carcinoma. Gynecol Oncol 104: 217-221, 2007.

72. Ogawa M, Ikeuchi K, Watanabe M, Etoh K, Kobayashi T, Takao Y, Anazawa S and Yamazaki Y: Expression of matrix metalloproteinase 7, laminin and type IV collagen-associated liver metastasis in human colorectal cancer: Immunohistochemical approach. Hepatogastroenterology 52: 875-880, 2005.

73. Zheng H, Takahashi H, Murai Y, Cui Z, Nomoto K, Niwa H, Tsuneyama K and Takano Y: Expressions of MMP-2, MMP-9 and VEGF are closely linked to growth, invasion, metastasis and angiogenesis of gastric carcinoma. Anticancer Res 26 3579-3583, 2006.

74. Wu CY, Wu MS, Chiang EP, Chen YJ, Chen CJ, Chi NH, Shih YT, Chen GH and Lin JT: Plasma matrix metalloproteinase-9 level is better than serum matrix metalloproteinase-9 level to predict gastric cancer evolution. Clin Cancer Res 13: 2054-2060, 2007.

75. Mylona E, Nomikos A, Magkou C, Kamberou M, Papassideri I Keramopoulos A and Nakopoulou L: The clinicopathological and prognostic significance of membrane type 1 matrix metalloproteinase (MT1-MMP) and MMP-9 according to their localization in invasive breast carcinoma. Histopathology 50: 338-347, 2007.

76. Yang YH, Deng H, Li WM, Zhang QY, Hu XT, Xiao B, Zhu HH, Geng PL and Lu YY: Identification of matrix metalloproteinase 11 as a predictive tumor marker in serum based on gene expression profiling. Clin Cancer Res: 14: 74-81, 2008.
77. Cheng CW, Yu JC, Wang HW, Huang CS, Shieh JC, Fu YP, Chang CW, Wu PE and Shen CY: The clinical implications of MMP-11 and CK-20 expression in human breast cancer. Clin Chim Acta 411: 234-241, 2010.

78. Jones LE, Humphreys MJ, Campbell F, Neoptolemos JP and Boyd MT: Comprehensive analysis of matrix metalloproteinase and tissue inhibitor expression in pancreatic cancer: increased expression of matrix metalloproteinase-7 predicts poor survival. Clin Cancer Res 10: 2832-2845, 2004

79. Thewes M, Pohlmann G, Atkinson M, Mueller J, Pütz B and Höfler H: Stromelysin-3 (ST-3) mRNA expression in colorectal carcinomas. Localization and clinicopathologic correlations. Diagn Mol Pathol 5: 284-290, 1996.

80. Ito Y, Yoshida H, Kakudo K, Nakamura Y, Kuma K and Miyauchi A: Inverse relationships between the expression of MMP-7 and MMP-11 and predictors of poor prognosis of papillary thyroid carcinoma. Pathology 38: 421-425, 2006.

81. Mellick AS, Blackmore D, Weinstein SR and Griffiths LR: An assessment of MMP and TIMP gene expression in cell lines and stroma - tumour differences in microdissected breast cancer biopsies. Tumour Biol 24: 258-270, 2003.

82. Têtu B, Trudel D and Wang CS: Proteases by reactive stromal cells in cancer: An attractive therapeutic target. Bull Cancer 93: 944-948, 2006 (In French).

83. Hsin CH, Chen MK, Tang CH, Lin HP, Chou MY, Lin CW and Yang SF: High level of plasma matrix metalloproteinase-11 is associated with clinicopathological characteristics in patients with oral squamous cell carcinoma. PLoS One 9: e113129, 2014.

84. Chang WJ, Du Y, Zhao X, Ma LY and Cao GW: Inflammationrelated factors predicting prognosis of gastric cancer. World $\mathrm{J}$ Gastroenterol 20: 4586-4596, 2014.

85. Pedersen G, Saermark T, Kirkegaard T and Brynskov J: Spontaneous and cytokine induced expression and activity of matrix metalloproteinases in human colonic epithelium. Clin Exp Immunol 155: 257-265, 2009.

86. Xu CJ and Xu F: MMP-11 and VEGF-C expression correlate with clinical features of colorectal adenocarcinoma. Int J Clin Exp Med 7: 2883-2888, 2014

87. Eiró N, Fernandez-Garcia B, Vázquez J, Del Casar JM, González LO and Vizoso FJ: A phenotype from tumor stroma based on the expression of metalloproteases and their inhibitors, associated with prognosis in breast cancer. Oncoimmunology 4: e992222, 2015.

88. Mari BP, Anderson IC, Mari SE, Ning Y, Lutz Y, Kobzik L and Shipp MA: Stromelysin-3 is induced in tumor/stroma cocultures and inactivated via a tumor-specific and basic fibroblast growth factor-dependent mechanism. J Biol Chem 273: 618-626, 1998.

89. Peruzzi D, Mori F, Conforti A, Lazzaro D, De Rinaldis E, Ciliberto G, La Monica N and Aurisicchio L: MMP11: a novel target antigen for cancer immunotherapy. Clin Cancer Res 15: 4104-4113, 2009.

90. Hofmeister V, Schrama D and Becker JC: Anti-cancer therapies targeting the tumor stroma. Cancer Immunol Immunother 57: $1-17,2008$.

91. Yi T, Wei YQ, Tian L, Zhao X, Li J, Deng HX, Wen YJ, Zou CH, Tan GH, Kan B, et al: Humoral and cellular immunity induced by tumor cell vaccine based on the chicken xenogeneic homologous matrix metalloproteinase-2. Cancer Gene Ther 14: 158-164, 2007.

92. Yokoyama Y, Grünebach F, Schmidt SM, Lazzaro D, De Rinaldis E, Ciliberto G, La Monica N and Aurisicchio L: Matrilysin (MMP-7) is a novel broadly expressed tumor antigen recognized by antigen-specific $\mathrm{T}$ cells. Clin Cancer Res 14: 5503-5511, 2008

93. Groves MD, Puduvalli VK, Hess KR, Jaeckle KA, Peterson P, Yung WK and Levin VA: Phase II trial of temozolomide plus the matrix metalloproteinase inhibitor, marimastat, in recurrent and progressive glioblastoma multiforme. J Clin Oncol 20: 1383-1388, 2002. 